\title{
Cluster protocols in Underwater Sensor Networks: a Research Review
}

\author{
K. Ovaliadis" and N. Savage \\ School of Engineering and School of Computing, University of Portsmouth, Portsmouth, UK
}

Received 13 June 2014; Accepted 5 July 2014

\begin{abstract}
Underwater Wireless Sensor Networks (UWSN) have different characteristics in relation to terrestrial wireless sensor networks; such as limited bandwidth capacity, high propagation delays and limited battery power. Although there has been much work completed in developing protocols and models for terrestrial networks, these are rarely applicable for underwater sensor networks. Up to today major efforts have been made for designing efficient protocols while considering the underwater communication characteristics. An important issue on this research area is the construction of an efficient clustering algorithm. Clustering in the context of UWSN is important as it contributes a great deal towards the efficient use of energy resources. This paper reviews the most significant cluster based protocols proposed for UWSN. Major performance issues of these protocols with respect to the network conditions such as packet delivery ratio, average packet delay, node mobility effect and energy consumption are examined. The advantages and disadvantages of each protocol are also pointed out.
\end{abstract}

Keywords: Underwater wireless sensor networks, cluster protocols, energy efficiency, routing.

\section{Introduction}

Energy consumption is an important issue during the design and the overall performance evaluation of an UWSN system. One main factor that affects this performance is that sensors are typically using batteries as the only power source. This is the main reason researchers in this area are focusing on designing more scalable and energy efficient routing protocols [1]. The challenges are many and they have to be overcome in order to achieve practical and cost effective solutions. Such challenges include the long latency and limited bandwidth, the noise, the high bit error rates, the transmission loss, the reliability issues, the energy and connection quality [2][3].

Most researchers concentrate on the individual sensor node energy-efficiency behaviour and they have proposed a number of underwater routing protocols such as DepthBased Routing (DBR) [4], Vector-Based Forwarding (VBF) [5], Hop-by-Hop Vector-Based Forwarding (HH-VBF) [6], Sector Based Routing with Destination Location Prediction (SBR-DLP) [7], Focused Beam Routing (FBR) [8], and Under-Water Diffusion (UWD) [9].

Some others came up with an underwater sensor network architecture that considers the energy-efficiency clustering routing protocols offer. Clustering schemes give a major effort to reduce the energy consumption in order to prolong the network lifetime. The most recently proposed clustering protocols for UWSN include the Distributed Underwater Clustering Scheme (DUCS) proposed by Domingo and Prior [10] the Multipath Virtual Sink (MVS) routing protocol architecture proposed by Seah and Tan [11], the distributed Minimum-Cost Clustering Protocol (MCCP) proposed by $\mathrm{Pu}$

\footnotetext{
*E-mail address: Kyriakos.0valiadis@port.ac.uk ISSN: (C) 2014 Kavala Institute of Technology. All rights reserved.
}

et al. [12], the Temporary Cluster Based Routing (TCBR) algorithm proposed by Ayaz et al. [13], the hydraulic pressure routing protocol, Hydrocast, proposed by Uichin et al. [14] and the Location-based Clustering Algorithm for Data Gathering (LCAD) proposed by Anupama et al. [15].

This article investigates, in more depth, UWSN cluster protocols in order to highlight their contribution towards the concept of energy efficient networks. In section two the performance of these cluster protocols is presented and evaluated leading to a critical analysis and discussion in section three.

\section{Cluster based protocols review}

\subsection{Distributed underwater clustering scheme (DUCS)}

DUCS [10] is a new addition of a distributed energy-aware routing protocol. It specifically designed for long-term nontime-critical aquatic monitoring applications using UWSNs with random node mobility and without requiring the assistance of a GPS. According to the authors, DUCS offers flexibility, does not use flooding techniques, minimizes the proactive routing message exchange and it uses data aggregation to reduce the redundant information to the sinks. During the operation of the protocol the network sensors are organised into clusters. A given sensor node acts as the cluster head $(\mathrm{CH})$ and all the remaining nodes send data packets to their corresponding $\mathrm{CH}$ with a single hop. Once the cluster head receives the data from all these sensor nodes, it carries out signal processing operations such as aggregation. Then it sends the aggregate data to the sink through other cluster heads by using a multi-hop routing.

According to the DUCS model (see Fig. 1) cluster heads are not only in charge of organising the members of their cluster (intra-cluster coordination) but also they are 
responsible for establishing communication between different clusters of the entire network (inter-cluster communication). Furthermore, the cluster head is entirely selected using a randomized rotation method amongst nodes of the same cluster team, minimizing in this way the fast depletion of the battery energy.



Fig 1. A network case using DUCS.

DUCS performs the energy operation in two stages. The first stage involves the setup process where the network is split into clusters. In the second stage several frames are transmitted to each cluster head where every frame is composed of a series of data messages that the ordinary sensor nodes send to the cluster head with a schedule.

Evidence provided by the authors based on simulation, suggests that DUCS manages to offer both a high ratio of packet delivery and is energy efficient. However, the designers have also reported a number of performance issues. They have observed that node movements can impact the cluster structure and this in turn can minimize the cluster's life expectancy. Organizing the network sectors continuously leads to an energy inefficient network. Another issue highlighted is that cluster heads can also move due to the environmental conditions and this probably could terminate the communication link even if few non-cluster head nodes are available between them.

\subsection{Multipath Virtual Sink (MVS)}

In this routing protocol architecture [11], the entire network is also divided into clusters following an innovative approach where each cluster has one or many local aggregation points. These aggregation points form a small mesh network that connects to local sinks as shown in Figure 2.

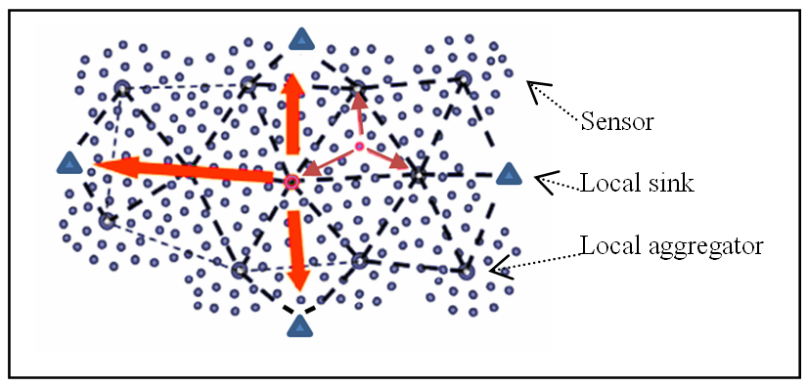

Fig 2. Multipath virtual sink topology.

The underlying assumption in this architecture is that local sinks are connected to each other using high speed communication channels, such as RF, forming a virtual sink. As the acoustic channel presents connectivity irregularities and thus available bandwidths are very small, the sensor nodes will be more functional if they cache the collected data and then send it when the channel conditions are optimum. In the case of delayed data, the proposed network architecture will attempt to send data packets using multiple paths; thus improving the conditions for successful data delivery.

The local aggregation points create a wireless mesh network in which multiple local sinks can be reached through multiple paths. The formation of the multiple paths is performed during an initialization phase. In this phase, every sink node broadcasts a hop count update packet to make its identity known. All the sensor nodes receiving this packet update their hop-count value, and then rebroadcast the packet incrementing the count by one. When a sensor node has data for sending, it can forward this packet towards any linked local sink using the previous hop recursively. The performance of the MVS architecture is assessed by carrying out many transmissions with a single path and then forwarding multiple copies along different routes in order to secure that the transmissions are getting through to different sinks. As long as a copy of the packet reaches one of these sinks, delivery is successful. The use of spatially diverse paths reduces the latency, the number of packet transmissions and the possibility of inter-path contention. In conclusion reliability is improved as duplicate packets are delivered towards multiple sinks through multiple paths. However, the problem of redundant transmission exists and this is amplified by the nodes' movement leading to more energy consumption.

\subsection{Distributed minimum-cost clustering protocol (MCCP)}

$\mathrm{Pu}$ et al. [12] proposed a distributed minimum-cost clustering protocol (MCCP) aiming to achieve energy efficiency and extension of the network's life expectancy. The suggested model takes advantage of a cluster driven approach where clusters are formed by taking into consideration three parameters; the total energy required by the cluster members for sending data to the cluster head, the residual energy of the cluster head and its entire members, and the relative location of the cluster head and underwatersink (uw-sink).

The first step of this approach is to select clusters based on a centralized algorithm called minimum-cost clustering algorithm (MCCA). The MCCA algorithm is then extended to the above mentioned distributed approach called MCCP. Initially in this scheme all sensor nodes are candidates, eligible to construct their neighbour set and their uncovered neighbour set. At this point it must be noted that the last set includes the nodes that are in the neighbour set but are still candidates. Then every candidate searches every possible combination of elements in its uncovered neighbour set; generating in this way its potential clusters. Thereafter it selects a representative from these clusters, of which the average cost is estimated and then broadcasted with its cluster head ID to all candidate nodes in a range of 2-hops. Once the cost has been calculated, every candidate node will compare it with its own calculated cost. In the case the candidate node has minimum average cost then it becomes a cluster-head and sends an INVITE to the other nodes to join the cluster as members. On the other hand if the sending node has better cluster cost, the receiving node extracts the cluster head ID from the received packet and sends a JOIN message. Once the clusters have been formed, a TDMA 
schedule is defined and be sent to the respective cluster members.

This approach is eliminating the formation of hot spots around the uw-sink by creating more cluster heads balancing in this way the traffic flow. The locations of the cluster head and the uw-sink define the number of the cluster members. This implies that clusters near the uw-sink will have less cluster members. In addition, this scheme is capable of balancing the traffic flow by rearranging the cluster nodes according to the minimum average cost principle. However the constant movement of sensors may increase the reclustering period, affecting the cluster and energy efficiency.

\subsection{Temporary Cluster Based Routing (TCBR)}

Most of the proposed multi-hop routing protocols encounter a major problem with the nodes near to the sink. These sensors handle a major load of information that forces them to use more power and therefore to drain their energy earlier comparing to the other sensors. In order to solve this problem and make energy consumption more equal throughout the network, Ayaz et al. [13] proposed a Temporary Cluster Based Routing (TCBR) algorithm. In the TCBR architecture, multiple sinks are deployed inside the water area and any packets received by them are considered successfully delivered. This is accomplished due to sink's ability to use radio communication which leads to a small propagation delay.

Two different kinds of nodes are used: ordinary sensors and some special sensors called courier nodes. Ordinary sensors are used to sense an event, collect the useful information and try to forward these data to a nearer courier sensor node. Courier sensors also assume the responsibility of delivering these data packets (taken from the other ordinary sensor nodes) to a surface sink. One of the goals of this architecture is that only a small number of courier nodes ( $2-4 \%$ of total sensor nodes) are used. Every courier node is assembled with a mechanical module, a piston, which has the ability to create positive and negative buoyancy. This module actually helps the nodes to move inside the water at different predefined depths and then pull them back to the sea surface. Every time these nodes reach different depth levels, they stop for a specified period and they broadcast hello packets to discover any ordinary nodes around them. Hello packets can be forwarded only within 4 hops. If an ordinary node receives more than one of these then it will forward the data packet to the nearer courier node, defined in the hello packet, within a specified amount of time. The analysis of this algorithm shows not all sensors are mobile but only the courier nodes due to their mechanical part.

The equal energy consumption task is completed by this algorithm throughout the network requiring only a small number of courier nodes, instead of equipping every sensor with the mechanical module. However, data can be only gathered when a courier node is located inside the communication range of every sensor node. Because of this, all the sensor nodes will keep their data packets in a limited buffer until a courier node reaches them. Despite this feature, the TCBR is not suitable for time critical applications [16].

\subsection{HydroCast}

Uichin et al. [14] proposed a hydraulic pressure based anycast routing protocol called HydroCast in order to overcome the limitations of geographic routing. This algorithm uses the measured pressure levels to find the routes for forwarding packets from source to the surface buoys. It is stateless and completes its task without requiring expensive distributed localization. Hydrocast nodes are equipped with a low cost pressure sensor to measure their own depth locally. Multiple mobile sinks are also deployed on water surface, which move with water flow. With regard to discovering a positive progress area toward to the sink, this protocol exploits only the information that is estimated by measuring the pressure of water in different depths.

The proposed scheme has two stages; the forward selection set and the routing recovery mode. In the first stage an opportunistic forwarding mechanism is used to select a subset (cluster) of neighbouring nodes with higher progress toward to the sink as the next hop candidates. The neighbouring nodes that receive a packet will access their priority according to their distance to the destination; the closer to the destination the higher priority. In this subset a node will forward the packet only when all nodes with higher priority progress to the destination fail to send it. This process is scheduled with the use of a back-off timer which is set up proportional to the destination's distance. All the other sensors with lower priorities will suppress their transmissions upon receiving the transmission (data or ACK packet) of a higher priority node. By this way the possibility of collisions and redundant transmissions is minimized.

In the second stage, a local maximum recovery mechanism is introduced in order to deal with the communication void. A node is considered as a local maximum node if there are no neighbours with lower pressure levels. To overcome this problem it enables a void handling mechanism. According to this, each local maximum node finds and stores a recovery path to a node whose depth is lower than itself and transmits the data packet to this node.

\subsection{Location-based Clustering Algorithm for Data Gathering (LCAD)}

Anupama et al. [15] suggested a clustering algorithm based on the geographical location of the sensor nodes in a 3-D hierarchical network architecture in order to extend the lifetime of the network.

In this architecture the sensor nodes are deployed in the area of interest at fixed relative depths from each other. Then all sensors at each tier are organized in clusters with multiple cluster heads. The selection of the cluster head follows a specific algorithm that takes into account the position of the sensors in the cluster.

The intra-cluster communication between sensor nodes is carried out using horizontal acoustic links and the intercluster communication between cluster heads is using vertical links. The length of these vertical acoustic links is limited to $500 \mathrm{~m}$. This restriction is based on estimations that show that acoustic links with distances less than $500 \mathrm{~m}$ can have optimal performance [17].

In the proposed architecture, the entire network is divided into three-dimensional grids. Each grid is set approximately to $30 \mathrm{~m} * 40 \mathrm{~m} * 500 \mathrm{~m}$. The communication process is carried out in three stages:

i. the setting up stage that includes the selection of the cluster head,

ii. the data collection stage, where the cluster head collects the data send it by the nodes in the same cluster and,

iii. the transmission stage, where the gathered data is transmitted from each cluster head to the base 
station with the assistance of Autonomous Underwater Vehicles (AUVs) [18].

In addition, sensor nodes that have some extra features such as more memory and energy resources are qualified to become $\mathrm{CH}$-heads. The outcome of having multiple $\mathrm{CH}$ nodes is the increase of the reliability and the load balancing in the network. The location of these $\mathrm{CH}$ nodes which is approximately at the centre of the grid helps the communication with the maximum number of ordinary sensor nodes. It can be stated that the arrangement of the grid takes the form similar to a cellular network.

According to the authors the biggest disadvantage of this approach is that LCAD performance is associated with the grid organization and in particular is dependent on the position of the $\mathrm{CH}$-node. This becomes more of an issue when the approach is implemented in an underwater environment where node movements cannot be avoided and as a result grid structure becomes more unstable. The performance analysis of this approach was carried out in a static environment and it has been tested in terms of energy consumption without taking into consideration the underwater conditions of the actual environment.

\section{Critical assessment and discussion}

All papers reviewed in this study evaluate the performance of their cluster protocols on the basis of the mechanisms employed by each protocol i.e. RTS/CTS, holding time, probability of collision etc.[16,19,20,21]. Based on their assessment this critical review present a comparison of all algorithms as they have been assessed by their respective research for each of them. To evaluate the performance of the above discussed protocols, this study adopted a weighting scale from one (1) to three (3) where one is low, two is average and three is high. A number of criteria as shown in the vertical row of table 1 have been inserted for this comparison. The finding indicates that the Hydrocast with 14 points comes on top with a small difference from the Multipath Virtual Sink cluster protocol. The remaining protocols average between 9 and 11 thus they can be classified as offering approximately the same performance. However, in terms of energy efficiency MCCP is the only one that scores high.

\section{Conclusion}

One of the main goals of clustering is the traffic load balance between cluster heads and cluster members [19]. This can be accomplished by periodically re-clustering the sensor nodes in the network. However since the cost of the re-clustering influence the protocols' performance, the period of this process requires further, in depth consideration. Instead of a fixed period of re-clustering, an adaptive criterion can be used. For example a re-clustering period can be taken into account based on the mobility of the nodes or the number of redundant transmissions.

Another issue of these algorithms' performance is that they depend on the device discovery time, i.e., the time taken by a node to discover and to connect to another node in its range. For an efficient clustering algorithm the time taken to complete the formation of the cluster team is very crucial, especially when the number of sensors is large. Delay in the initial phase of clustering means more packet transmissions and more power consumption.

An important subject that also needs further investigation is the effect of adding more sensors into the network system after the initial clustering phase. Many questions come up such as; is it necessary for the system to re-cluster in order to find these new sensors? If yes then how soon the system will be in position to start the re-cluster process?

The results indicate that the overall performance of the HydroCast cluster protocol dominates the others. However, the MCCP comes top in the energy efficiency area. It could be suggested that a way could be found to incorporate the energy efficiency features of the MCCP into HydroCast in order to develop a better performance cluster algorithm.

Table 1. Performance characteristics of the selected protocols.

\begin{tabular}{|c|c|c|c|c|c|c|c|}
\hline Protocol & $\begin{array}{l}\text { Delivery } \\
\text { ratio }\end{array}$ & $\begin{array}{l}\text { Energy } \\
\text { efficiency }\end{array}$ & \begin{tabular}{|l|}
$\begin{array}{l}\text { Delay } \\
\text { efficiency }\end{array}$ \\
\end{tabular} & $\begin{array}{l}\text { Bandwidth } \\
\text { efficiency }\end{array}$ & Reliability & $\begin{array}{l}\text { Mobility } \\
\text { efficiency }\end{array}$ & \begin{tabular}{|l} 
Performance \\
Score \\
\end{tabular} \\
\hline DUCS & average & \begin{tabular}{|l|} 
average \\
\end{tabular} & low & average & low & average & 10 \\
\hline $\begin{array}{l}\text { Multipath } \\
\text { Virtual Sink }\end{array}$ & average & low & average & average & High & average & 13 \\
\hline MCCP & low & high & low & average & average & low & 10 \\
\hline TCBR & average & average & low & average & average & low & 10 \\
\hline HydroCast & high & average & high & average & average & average & 14 \\
\hline LCAD & average & average & low & average & low & low & 9 \\
\hline
\end{tabular}




\section{References}

[1] M. C. Domingo and R. Prior, "Energy analysis of routing protocols for underwater wireless sensor networks", Computer Communications, vol.31, Issue 6, pp. 1227-1238, April 2008.

[2] I. F. Akyildiz, D. Pompili, and T. Melodia, "Underwater Acoustic Sensor Networks: Research Challenges", Journal of Ad Hoc Networks, vol. 3(3), pp. 257-279, Elsevier, Amsterdam, May 2005.

[3] J. Heidemann, Y. Li, A. Syed, J. Wills, and W. Ye., "Underwater sensor networking: Research challenges and potential applications", in Proc. Of the IEEE Wireless Communications and Networking Conference, Las Vegas, Nevad, USA, April 2006.

[4] Hai Yan, Zhijie Shi, Jun-Hong Cui, "DBR: Depth based Routing for Underwater Sensor Networks", Springerlink NETWORKING, pp.16-1221, 2008.

[5] P. Xie, JH. Cui, L. Lao, "VBF: vector-based forwarding protocol for Underwater Sensor Networks", IFIP NETWORKING, pp. 1216$1221,2006$.

[6] Nicolas Nicolaou, Andrew See, Peng Xie, "Improving the robustness of location-based routing for Underwater Sensor networks", IEEE OCEANS 2007, pp. 1-6, 2007.

[7] N. Chirdchoo, Wee-Seng Soh; Kee Chaing Chua, "Sector-Based Routing with Destination Location Prediction for Underwater Mobile Networks", IEEE international conference on Advanced Information Networking and Applications WAINA'09, pp. 1148$1153,2009$.

[8] M. Jornet, M. Stojanovic, M. Zorzi, "Focused beam routing protocol for underwater acoustic networks", 3rd ACM conference WuWNet'08, 2008.

[9] U. Lee, J. Kong, S. J Park, E. Magistretti, and M. Gerla, "Timecritical underwater sensor diffusion with no proactive exchanges and negligible reactive floods", in: Proceedings 11th IEEE Symposium on Computers and Communications (ISCC'06), pp. 609-615, 2006.

[10] M.C. Domingo, R. Prior, "A distributed clustering scheme for underwater wireless sensor networks", in: Proceedings of 18th Annual IEEE International Symposium on Personal, Indoor and Radio Communications (PIMRC 2007), pp. 1-5, 2007.

[11] Winstonm K.G. Seah, Tan Hwee-Xian, "Multipath Virtual Sink Architecture for Underwater Sensor Networks", IEEE OCEANS 2006, pp. 1-6, 2006.

[12] Pu Wang, Cheng Li, Jun Zheng, "Distributed Minimum-Cost Clustering Protocol for UnderWater Sensor Networks (UWSN)", IEEE International Conference on Communications ICC'07, pp. 3510- 3515, 2007.

[13] M. Ayaz, A. Abdullah, J. Low Tang, "Temporary cluster based routing for Underwater Wireless sensor Networks", in: Proceedings of the International Symposium in Information Technology (ITSim), pp. 1009 - 1014, 2010.

[14] Uichin Lee, Paul Wang, Youngtae Noh, Luiz F.M.Vieira, Mario Gerla, Jun-Hong Cui, “ Pressure Routing for Underwater Sensor Networks", IEEE INFOCOM, pp. 1-9, 2010.

[15] KR. Anupama, A. Sasidharan, S. Vadlamani, "A location-based clustering algorithm for data gathering in $3 \mathrm{D}$ underwater wireless sensor networks", in: Proceedings of the International Symposium on Telecommunications, IST, pp. 343-348, 2008.

[16] M. Ayaz, I. Baig, A. Azween, and F. Ibrahima, "A survey on routing techniques in underwater wireless sensor networks," Journal of Network and Computer Applications, 2011.

[17] M. Stojanovic, JA. Catipovic, JG. Proakis, "Phase-coherent digital communications for underwater acoustic channels" IEEE J. Oceanic Eng., pp. 100-11, 1994.

[18] M. Erol, L. Vieira, M. Gerla, "AUV-aided localization for underwater sensor networks", in: Proceedings of International Conference on Wireless Algorithms, Systems and Applications (WASA), pp. 97-100, 2007.

[19] Abdul Wahid, Kim Dongkyun, “Analyzing Routing Protocols for Underwater Wireless Sensor Networks ", International Journal of Communication Networks and Information Security (IJCNIS), vol. 2, no. 3, pp. 253-261,2010.

[20] C.Giantsis and A.A. Economides, "Comparison of routing protocols for underwater sensor networks: a survey," International Journal of Communication Networks and Distributed Systems, vol. 7, no. 3-4, pp. 192-228, 2011.

[21] M. T. Kheirabadi and M. M. Mohamad, "Greedy routing in underwater acoustic sensor networks: a survey," International
Journal of Distributed Sensor Networks, vol. 2013, Article ID 701834, 21 pages, 2013. 